\title{
Synergistic Allocation of Flight Expertise on the Flight Deck (SAFEdeck): A design concept to combat mode confusion, complacency, and skill loss in the flight deck
}

\author{
Paul Schutte ${ }^{1}$, Kenneth Goodrich ${ }^{1}$, Ralph Williams ${ }^{2}$ \\ ${ }^{1}$ NASA Langley Research Center, Hampton, Virginia, 23681, USA \\ ${ }^{2}$ Analytical Mechanics Associates, Hampton, VA, 23666, USA \\ \{Paul.C.Schutte, Kenneth.H.Goodrich, Ralph.A.Williams\}@nasa.gov
}

\begin{abstract}
This paper presents a new design and function allocation philosophy between pilots and automation that seeks to support the human in mitigating innate weaknesses (e.g., memory, vigilance) while enhancing their strengths (e.g., adaptability, resourcefulness). In this new allocation strategy, called Synergistic Allocation of Flight Expertise in the Flight Deck (SAFEdeck), the automation and the human provide complementary support and backup for each other. Automation is designed to be compliant with the practices of Crew Resource Management. The human takes a more active role in the normal operation of the aircraft without adversely increasing workload over the current automation paradigm. This designed involvement encourages the pilot to be engaged and ready to respond to unexpected situations. As such, the human may be less prone to error than the current automation paradigm.
\end{abstract}

Keywords: Human Factors · Human-systems Integration · Function Allocation · Flight Deck · Aviation · Human Error · Skill Loss

\section{Introduction}

The vision of a future flight deck for civilian and military aircraft is often one of no flight deck at all. Phrases such as 'Increasing Automation' and 'Automation Autonomy' dominate many research and development programs within NASA and the Department of Defense. The vision of uncrewed aircraft is one of increased efficiency, precision, and reliability and reduced costs and errors. Humans are often considered a liability to the system. The rationale for full automation is straightforward. Most accidents are found to be caused by human error. The human must be the weak link in the chain. Since automation has hardly ever been found at fault for causing an accident, more automation plus less human equals greater safety.

Unfortunately, this does not appear to be the case. The modern civil aircraft is highly automated and the human's role has decreased substantially [1]. And yet, these aircraft are still involved in accidents. The fact that human errors are still named as the causes 
of these accidents gives rise to the question: Why hasn't the reduction in human involvement in the operation of the aircraft resulted in a commensurate reduction in accidents caused by human error?

Similar questions have been raised regarding the issue of pilot workload in the flight deck. Since much of what the pilot used to perform has been allocated to the automation, why isn't there a significant decrease in the pilot's perceived workload? This question has been answered. Studies have shown that while the pilot's physical workload decreased in highly automated aircraft, their mental workload increased. Automation did not reduce pilot workload; it simply changed the nature of that workload $[2,3,4]$.

Perhaps the fact that human error and workload have not appreciably decreased in the modern flight deck is due, in part, to how the automation has been implemented as opposed to the amount of automation in the flight deck. There has been much research demonstrating how humans have fared poorly with increased automation; for example, automation complacency, overreliance on automation, loss of situational awareness and spatial orientation, and skill loss. These have contributed to human errors.

This paper presents a new flight deck design based on a function allocation approach called Complemation [5]. Complemation focuses on the role of the human in the flight deck; specifically, 'Why must the human be in the flight deck?' It uses automation and design to surround and support the human in performing that role. This is in contrast to substitution-based and machine-based forms of function allocation [6]. Substitution based function allocation considers all the tasks that have to be performed in the flight deck and determines whether the human or the automation can perform them better. The winner is given the task. Machine-based allocation operates under the assumption that machines are inherently better than humans and that the design should use automation to its fullest. In machine-based allocation schemes, the human is assigned the 'leftovers,' that is, the tasks that automation cannot handle. But these tasks are usually very difficult and can be disparate and non-cohesive from the human's perspective. In Complemation, some tasks may be allocated to the human even though the automation may be able to perform some aspects of that task better than the human. Automation is used purposefully and deliberately as opposed to wherever it can be used.

The flight deck design produced by this approach is called the Synergistic Allocation of Flight Expertise in the Flight Deck (SAFEdeck). It has many commonalities with current flight deck designs and concepts but there are significant differences. One big difference is that the flight automation is controlled using the active feedback control inceptors (e.g., stick and throttle) rather than using an autopilot interface on the glare shield and a Flight Management System (FMS) Control and Display Unit (CDU). The inceptors are the only way in which the pilot can command the aircraft to move. The pilot is more actively involved in the progress of the flight. The automation supports the pilot by actively engaging and managing their attention so they do not forget to perform tasks and adhere to flight restrictions.

The first section of this paper sets the stage for describing SAFEdeck by providing an analogy using automobile driving. The next section constitutes the bulk of the paper where the SAFEdeck design is described. This section also concludes with a summary of results from an experiment that investigated this instantiation. The last section includes suggestions for future work and for implementation of the function allocation strategy. 


\section{An automobile analogy}

Like aircraft operations, the SAFEdeck concept is extremely complex and detailed. As such, it is impossible to fully describe the concept in a paper such as this. In order to aid the reader in understanding SAFEdeck, an analogy may be beneficial. The analogy is based on automobile driving using advanced technology.

The Drive. A driver wants to travel from New York City to Orlando, Florida. The first step is to create a route using a route planner (e.g., Goggle Maps ${ }^{\mathrm{TM}}$, Garmin ${ }^{\mathrm{TM}}$, Tom $\mathrm{Tom}^{\mathrm{TM}}$ ). The planner uses published highway and roadway data to create the route. The driver can modify this route as needed. The route is loaded into the automobile's navigation system. The driver then begins the trip. Navigation information is provided on the driver's heads up display, as well as a top-down map display. Both displays depict the roadways and highways (even if they are not on the planned route.)

The driver's first goal is to get to the interstate. There are several predetermined courses to get from the driver's house to the interstate (each course is a different packaged or chunked path out of the city). The driver turns the car onto one of these courses. The automation recognizes this as a predetermined route and offers the driver the option to drive this route. The driver accepts and engages the automation with a trigger switch on the steering wheel. From that point, the automation will drive the car. The driver can take hands off the wheel and gas pedal.

While still in the city, a van breaks down in the road in front of the car. The automation interprets this as a traffic backup and waits. The driver sees that it is not a traffic backup and disconnects the automation. The driver puts on the turn signal to inform other drivers and the automation that they want to drive into the oncoming traffic lane to go around the van. The automation's monitoring looks for cars approaching from either direction and gives the driver a green indicator when it is safe to do so. The driver drives the car around the van and returns to the road. The automation asks if the driver wishes to resume the departure from the city to the interstate, the driver says yes, pulls the trigger and the automation resumes driving the car to the interstate.

As the car approaches the interstate, it informs the driver that the entrance is ahead. It is the driver's responsibility to disengage the automation and manually drive the car onto the interstate. Once on the interstate, the driver can turn complete control back over to the automation. If the driver wishes to go faster, they accelerate to the new speed and couple to that speed. When the car approaches a change in interstates (e.g., leaving I-95 to get on I-495), the automation notifies the driver that the ramp is coming up. The driver then disconnects the automation and merges on to the new interstate and then reconnects the automation. If the driver fails to disconnect and take the exit. The automation will provide louder and more alarming alerts. The car will remain on the interstate. However the warnings will continue until the driver actively silences them.

If the driver wishes to stop for the night at a hotel, the driver can select the hotel on the map display. The automation will alert the driver when the car is approaching the exit ramp. Again, the driver manually takes control and transitions to the local road. If the hotel is much farther down the road, the driver can tell the automation to follow the road and provide reminders when approaching the point where they need to leave the road. 
If the driver decides to simply drive around the countryside the next morning before returning to the interstate, the driver can drive on a road and then have the automation drive the car on that road. As the car approaches intersections, the automation alerts the driver of the intersection but nothing more. If the car comes to a $\mathrm{T}$ in the road where the driver must make a decision, the automation notifies the driver. If the car stops and the driver still has not intervened, the automation will sound the warning. If there is traffic behind the car, the automation will decide to turn one way or the other (to avoid obstructing traffic) but will pull over when able.

The Automation. Note that the car is not entirely self-driving. The driver does not enter a destination and then allow the car to independently drive all the way there. The car has limited automation, but that automation is extremely robust. The automation is responsible for all monitoring and for reminding the driver to make major transitions (e.g., home to city-exit course, city-exit course to interstate, interstate to interstate). But the driver must return to manual control to make those transitions. The automation will never willingly disconnect without the driver's approval. The automation can be overridden at any point.

The automation is aware of the road structure, the speed limits, facilities along the way, the weather conditions, and the plan. It is aware of its immediate surroundings (e.g., roads, other traffic, pedestrians and cyclists). It monitors road conditions. It is also aware of driver intervention and what the driver is doing (with regard to driving) even when the automation is not controlling the car. The logic of the automation has no high level reasoning skills and is entirely deterministic.

The Human. The human driver has the role of high level decision maker. If the driver is not situationally aware, they cannot perform this role and long durations of highly reliable automation can lead to complacency and distractibility. So the driver is called upon to periodically be part of the mission by making decisions at important junctures in the trip. It is unwise to expect that the driver will be paying attention otherwise.

The driver is also responsible for intervening in cases of automation failure or inability to appropriately perform. There may be cases where the automation doesn't know what to do or does not have authority. In these cases, the driver must return to manual control. If the driver has little or no regular experience with manual driving, there may be skill loss after a time. By requiring the driver to not only be involved in decision making at important junctures in the mission but also to manually drive, skill loss can be greatly reduced. Turns and decision points are more instructive in car handling skills than manual driving on a long stretch of highway.

\section{Synergistic Allocation of Flight Expertise in the Flight Deck (SAFEdeck)}

The SAFEdeck approach expands on the automobile system described above and applies it to aviation. The street/highway map is replaced with High and Low Altitude charts, Arrival, Departure, Approach and other terminal area charts and procedures. Nearly all of that information is contained in modern FMSs. The steering wheel is replaced by the active inceptors for the control surfaces and the gas pedal is replaced by 
an active throttle. The pilot has the role of high-level decision maker, risk manager and backup for the automation. Unlike today's aircraft, the entire mission may be planned, but execution of that mission requires human intervention at critical junctures. Unlike today's aircraft where there are three ways to control the aircraft (stick and throttle, autopilot/mode control panel (MCP), and FMS), there is only one way to control the aircraft and the automation - the active stick and throttle.

SAFEdeck is based on several design concepts, H-mode [7], the Naturalistic Flight Deck [8], and the Haptic Flight Control System [9]. The philosophy behind this approach is that one of the human's primary roles is to step in and deal with emergencies, non-normals, and highly complex or unanticipated situations. In some cases they must act as a backup for the automation or other resources. One of the goals of the design is to keep the pilot in the proper condition to perform these duties. To do this, SAFEdeck seeks to actively engage the pilot in the mission to maintain their situation awareness. This engagement will take the form of manually flying the aircraft at certain times in order to maintain skill level. In addition, the automation will conform to standard Crew Resource Management [10] principles as if it were another crew member. Finally, it is expected that the human will have deficiencies that lead to errors and the design must accommodate these deficiencies.

The SAFEdeck concept will be described by first defining the hardware components required, then the flight management functions and other functions critical to the concept. Finally, the results of an evaluation experiment will be briefly described.

\subsection{SAFEdeck Hardware Components}

There are many ways in which the SAFEdeck design can be implemented and so some of these descriptions will be deliberately vague. But the basic components either exist or are easily implemented in flight decks today. In many ways, the SAFEdeck flight deck will look very similar to modern flight decks. There are no dramatically new technology or display requirements. The main difference is how the automation and instrumentation is implemented, rather than the automation and instrumentation itself.

Active Inceptors. The two primary inceptors - the stick (or wheel and column) and the throttle - are active force-feedback inceptors. They are capable of transmitting haptic cues (such as pulses and vibrations) and are able to produce artificial force shaping to allow for resistance to envelope departures, detents, and other feedback signals. One force shaping feature is to allow virtual slots/tracks (similar to the slots/tracks in a standard transmission gear shifter pattern.) Each inceptor has at least three switches: A trigger for engaging the automation, a button for disengaging the automation, and a selection device (e.g., thumb wheel, hat-switch). The inceptor position always corresponds to the actual commands given to the control surfaces and engines.

Navigation Display. A large, easily accessible, high definition map display that can not only show the path that the aircraft is flying and waypoints, but also existing route structure and available options. The map should have a top-down perspective and a vertical perspective. Touch or cursor control will likely be a requirement. The Navigation Display is a primary instrument for normal flight and will likely be consulted as often as the Primary Flight Display. 
Primary Flight Display. This display contains the usual symbology found on modern primary flight displays. In addition a perspective view is presented behind the symbology. On this perspective view, not only is the current path portrayed in something like a highway in the sky, but also existing route structure. Waypoints in the form of 'waypoles' are also presented. Waypoles are vertical representations of waypoints. These waypoles may be flat earth representations if they are significantly far away.

Target Control Panel. Similar in many respects to modern autoflight interfaces (e.g., Mode Control Panels), the Target Control Panel allows the pilot to dial in specific headings, altitudes, airspeeds, and ascent/descent profiles. The major difference is that manipulating these parameters will not affect the aircraft's flight path. They merely create targets for the pilot to aim at / fly to. There may be additional parameter controls such as time of arrival or latitude/longitude.

Flight Planner. The Flight Planner is a separate device that is used to create plans, create what-if and alternate scenarios, and perhaps simulate the mission in fast time. The Flight Planner may be a portable device that communicates with the automation so that the pilot can make plans prior to flight or can use it as a tablet in flight, but this is not required. Ideally, the Navigation Display should not be used for the Flight Planner. The Navigation Display should always present real-time tactical information and it should not have additional clutter involved with the Flight Planner interface needs.

\subsection{SAFEdeck Flight Management Functions}

There are five basic Flight Management Functions in the SAFEdeck concept: Envelope Protection, Collision/Danger Avoidance, Self Preservation, Precision Assistance, and Active Flight Control. The first three are always on, however they can be overridden by the pilot. The last two assist the pilot in short-term, tactical maneuvers and control the aircraft over longer periods of time to manage workload and improve efficiency. They are used at the pilot's discretion.

Envelope Protection. This function impedes the ability to stall, overspeed, underspeed or barrel-roll the aircraft. When the aircraft is approaching one of these states, the pilot is alerted prior to this protection engaging. Before the aircraft actually enters one of these states, the inceptor will exert an artificial force that counteracts the condition. If the pilot does not intervene, this force will automatically return the aircraft to a safe orientation. The pilot can overpower this force and use the full capabilities of the aircraft. If the pilot releases the inceptor, the automation will seek to stabilize the aircraft

Collision/Danger Avoidance. This function serves to automatically avoid dangers such as other aircraft, severe weather, terrain, or restricted airspace. The pilot will be alerted as soon as possible so that they can avoid the danger themselves as they see fit. If the aircraft continues to advance towards the danger, the automation will increase the level of alert and it will provide artificial counter pressure on the inceptor to move away from the danger. Again, the pilot can override this feature.

Self Preservation. This function is used when the pilot is not responding due to incapacitation or impairment. If the pilot has not responded to an alert or failed to take control of the aircraft when the automation requests it, the automation will enter into 
self-preservation mode. In this mode, the automation emits an emergency transponder signal, and air traffic control (ATC) and all aircraft in the area are given notice that the automation is taking control of the aircraft on its own. The automation will then plan a route to the nearest acceptable airport and proceed to perform an automated emergency landing. ATC and the other aircraft are responsible for clearing the way for this aircraft as they would in any emergency.

As this is one of the few times the automation will make a mode change on its own and because it takes control away from the pilot, every effort will be made to ensure that the pilot can override this mode. It may be that the automation's first task is to descend to a breathable altitude in case the pilot is hypoxic. Additional safeguards may be necessary for this mode such as concurrence by ATC.

Precision Assistance. There are three aspects of this function. They may be thought of as a 'snap-to' feature, a 'restrict axis' feature, and a 'reset' feature.

Snap-To. If the aircraft is approaching a target (such as a heading that has been selected on the target control panel, or a published flight path or waypoint), and the pilot performs an action that appears to be trying to lock on to that target, the automation will home in on it so that the pilot does not have to struggle to make the precise corrections. The automation will stabilize on that target. It is important to note that the automation will not hold that target under precision assistance. If the pilot wants to hold to that target, they must use active flight control.

Restrict Axis. Often the pilot may wish to make a turn without changing altitude or may want to make an altitude change while staying on the lateral route (e.g., a jetway). The stick inceptor has a slight artificial force shaping in the form of two virtual slots forming a cross with the center of the cross located at the current position. If the pilot pulls directly back on the stick, they can feel the vertical slot created by the artificial force shaping. This will restrict movements to the vertical dimension only and will hold the lateral path constant. Likewise, if the pilot moves the stick to the left into horizontal slot, only lateral changes are made and the aircraft remains at the same altitude. It is important to note (yet difficult to describe) that the force shaping conforms to the actual pattern that would be required to maintain either axis. For example, when turning to the left, one might have to raise the nose slightly (pulling back on the stick) to maintain altitude. The slot will then bend slightly back to add this correction. This is due to a SAFEdeck constraint that the stick position always reflects what is happening in the aircraft. By shaping the alleyway to reflect real flight control corrections, the pilot always feels what should be done to maintain that axis. That way, if the precision assistance automation fails, the pilot will still be making the same stick movements that would be made by the automation. The pilot will never have to move the stick in one manner while the automation is active and another manner while the automation is disengaged.

Reset. This function is used to return the aircraft to a stabilized straight and level configuration. If the pilot finds themselves losing control of the aircraft, the pilot can call upon this function to have the automation right the aircraft. The pilot would use this feature if they become spatially disorientated. One possible implementation for this feature would be for the pilot to press and continue to hold the trigger on the stick. This appears congruent with the expected human physical response to disorientation - to 
grip the inceptor tightly. When in the reset mode, the stick inputs of the pilot are ignored $^{1}$.

Active Flight Control. In the automobile analogy offered in Section 2, the automation would couple to a road or highway and follow it without driver intervention. The SAFEdeck equivalent of this would be to couple to a jetway. The pilot would fly to the jetway, the automation would recognize it as something to follow, and the pilot would tell the automation to follow it. The airspace system is significantly more complicated than a country's road and highway system. SAFEdeck uses a category of objects called behaviors to handle the diversity of air travel. A jetway is a behavior. A holding pattern is a behavior. An approach is a behavior. A performance climb is a behavior. A takeoff is a behavior. A go-around is a behavior. A heading hold is a behavior. Behaviors are actions or sets of actions that the automation can perform autonomously. Behaviors can be published (e.g., jetways, approaches) or they can be created (e.g., heading hold, holding pattern, performance climb). In the automobile analogy above, the predetermined route out of the city would be a single behavior and is equivalent to a standard instrument departure from an airport.) Behaviors are generally geographically- based and have a start and an end - however they do not have to be (e.g., holding patterns can occur anywhere above a certain altitude and continue until the pilot decides to leave the pattern). The pilot can join a behavior at any point along its three dimensional path.

To couple to any behavior, the pilot performs the following:

- Fly the aircraft to the behavior and align it to the behavior

- Select the behavior (there may be more than one available at that location)

- Pull the trigger and engage the automation

Align, Select, Trigger is all the pilot has to remember to couple to a behavior. Precision assistance aids the pilot in aligning the aircraft to the behavior. In some cases it may be possible to create a behavior when you select it. For example, when the pilot points the aircraft at a waypoint, the automation gives the pilot the option to create a 'go-to' behavior to that waypoint. Pulling the trigger creates that behavior and couples the automation to it.

Disengaging the Automation. A dedicated button on the inceptors is used to disengage the automation. This is the preferred method. When the button is used to disengage an audible notification is given that a normal disengagement has taken place. Pressing the disengage button is the equivalent of telling the automation, "I have control of the airplane." The audible notification is the automation's way of saying that it concurs. Another way to disengage the automation is by force. If the pilot grabs the stick and provides a reasonable amount of pressure, the automation will disconnect and a caution alert will sound. This indicates that the automation was disengaged in a non-normal manner. Unless there is a failure, the automation will not automatically disengage. Following good Crew Resource Management [10] principles, it will not relinquish control until there is someone to receive it, thus ensuring that someone always has control of the aircraft.

\footnotetext{
${ }^{1}$ This is one of the few exceptions where the stick position may not agree with the actual control surface commands.
} 
Modifying a behavior. It is common for an aircraft to change altitude on a route due to weather or traffic. In such cases, the Restrict Axis precision assistance is used. Moving the stick either directly backwards or directly forwards so that it 'slides' into the artificial force alleyway will cause the automation to stay on the current lateral track of the behavior. Dialing an altitude into the Target Control Panel will create an altitude target. When the aircraft is approaching this altitude, the pilot receives a notification that it will be time to level off. When the pilot levels off near that altitude, the Snap-To precision assistance will home in on that altitude ${ }^{2}$.

At the Behavior's end. If the aircraft comes to the end of the behavior and the pilot has not transitioned to a new behavior or disconnected the automation, the automation will go into a safe state. A safe state varies depending on the type of behavior and if there is a plan in the system. At the end of an airway behavior, if there is a planned transition to another airway and the pilot has done nothing, the automation will make that transition on its own. However, this will constitute a warning that requires significant pilot input to silence. The significant input is to keep the pilot from becoming reliant on this feature (e.g., not bothering to disconnect, align, select, trigger because the automation will do it for them). If the pilot does not respond for the next behavior transition listed in the plan, the self-preservation mechanisms described above will engage. If there is no planned transition but there are published behaviors connected to the end of the current behavior, the automation will make an educated guess and pick one. If there are no planned or published transitions, the automation will transition to an altitude/heading hold behavior.

\subsection{SAFEdeck Notification and Alerting Functions}

SAFEdeck requires that the pilot must have a more interactive role in flying the aircraft and this includes making time- and position-critical inputs such as leveling off at the proper altitude. However vigilance and prospective memory (i.e., remembering to do something) are weak traits in human behavior [11]. It is vitally important that the SAFEdeck design includes a robust notification system that will ensure that the pilot remembers to intervene. Fortunately, vigilance and prospective memory are automation's strong suits. The SAFEdeck automation can provide notifications for:

- A behavior transition or parameter target is coming up (allowing the pilot to stop what they are doing and get back into the loop),

- It is time to make the behavior transition (e.g., disconnect, align, select, trigger),

- The pilot has failed to make the behavior transition, or

- The behavior has ended and the automation has gone into a safe state

These notifications increase in urgency, saliency, and alert level (Advisory, Caution, Warning). The goal is that the pilot will respond to the first two notifications in order to avoid the last two alerts.

\footnotetext{
${ }^{2}$ There is more detail to correctly achieving this procedure.
} 


\subsection{SAFEdeck Filtering and Decluttering Functions}

As mentioned previously, all published behaviors are presented on the navigation display. This is important because it allows the pilot to easily transition to an unplanned change/behavior by essentially flying to it and coupling the automation to the behavior instead of having to program the changes in a flight management system. However, there are far too many published airways, waypoints, arrivals, departures, and other procedures to present all of them on the navigation display. Another critical element of the SAFEdeck design is robust and efficient contextual behavior filtering and display decluttering. These functions would use context such as aircraft equipage, current plan, current altitude, phase of flight, direction, range, current airport information, and perhaps probability to filter out a significant amount of choices. Of course, the pilot should be able to select the filtering/decluttering methods so as to see more or fewer choices.

\subsection{Experiment Results}

The SAFEdeck concept increases the amount of physical workload on the pilot by bringing them back into the loop at junctures in the mission. The primary controls are now all routed through the 'stick and throttle' inceptors. These two factors have led to speculation that this is a step backwards to the flight decks of old rather than a step forward into the future. A part-task simulator study was performed to assess impact of the SAFEdeck concept on workload when compared to manual flying and fully automated flying. Additionally, the impacts on situational awareness, primary and secondary task performance, and subject preferences were assessed $[12,13]$.

Twenty-four high-time, non-instrument-rated pilots planned and flew four different flights in a fictitious airspace using a moderate-fidelity, part-task simulation. Each of the four runs was approximately one hour long. Three different flight control paradigms were tested: Manual Control (MC), Full Automation (FA) - a path-coupled automatic control typical of modern commercial aircraft, and a simplified version of the SAFEdeck concept. Subjects were required to make both tactical and strategic flight changes as well as perform two secondary tasks (target recognition and numeric calculation). An automation failure was introduced in the FA and the SAFEdeck conditions and the time to detect the failure was measured. Workload was measured using the NASA-TLX [14]. Situational awareness was measured using the SAGAT [15] protocol and subjective responses.

To summarize the statistically significant findings: 1) the SAFEdeck condition reduced Mental Demand and Effort when compared to the MC condition; 2) subjects detected a failure of the automation in the SAFEdeck condition sooner than they detected it in the FA condition; and 3) subjects preferred the SAFEdeck condition over both the FA and the MC conditions when considering just flying the aircraft and when considering flying the aircraft with secondary tasks.

The statistically significant results themselves are encouraging and they reinforce the claims of increased situation awareness, reduced workload, and high subject preference when using the SAFEdeck concept. While many of the results were not statistically significant, they all favored the SAFEdeck concept over the other two. 


\section{$4 \quad$ Summary}

SAFEdeck has six major features that make it unique from current automation strategies in the flight deck. The first combats mode confusion and skill loss and supports graceful degradation. SAFEdeck uses the manual control inceptors to manage and direct the automation rather than having the pilot use three uniquely different interfaces (the control inceptors, the autopilot (mode control panel) and the flight management system interface. The second feature addresses mode confusion and complacency while improving situational awareness. The pilot is involved in all major trajectory changes such as major heading and altitude changes. The third feature combats typical human errors that stem from forgetfulness. SAFEdeck takes advantage of automation's memory capacity and retrieval (retrospective, prospective, declarative, and procedural) to backup the pilot. A fourth feature addresses mode confusion as well as allowing more fluid tactical trajectory management support. It is the use of enhanced graphics on both the primary flight display and the map display that show all flight path options that are available and appropriate to the pilot. The fifth feature is the use of the automation as backup for human error, and the pilot as backup for the automation. This feature addresses problems of complacency and other types of human error. Finally, complacency and fatigue are addressed by imbuing the automation with 'self preservation' features that make it 'resistant but not insubordinate' to making blunders such as flight into terrain or continuing an unstable approach below a safe altitude.

SAFEdeck does not sacrifice efficiency or capability. The human automation team is as efficient and precise as today's flight management system/autopilot combination. All functions performed in the current automation scheme can be performed using the new paradigm. But it is much more natural and simplified to perform those functions using the SAFEdeck design approach. The SAFEdeck design can be implemented using today's technology and does not rely on advances in artificial intelligence, access to big data, or changes in the airspace system.

The next steps in research are to fully implement the SAFEdeck design and then perform usability studies on the design using pilots and non-pilots.

\section{$6 \quad$ References}

1. Aviation Safety Network http://aviation-safety.net/statistics/period/stats.php?cat=A1

2. Hart, S. G., \& Sheridan, T. B. (1984). Pilot workload, performance, and aircraft control automation. In Human factors considerations in high performance aircraft (AGARD-CP-371) (pp. 18/1-18/12). Neuilly Sur Seine, France: NATO-AGARD.

3. Wiener, E. L. (1988). Cockpit automation. In E. L. Wiener \& D. C. Nagel (Eds.), Human Factors in Aviation (pp. 433-461). San Diego: Academic Press.

4. Wiener, E. L. (1993). Life in the second decade of the glass cockpit. In R. S. Jensen \& D. Neumeister (Ed.), Proceedings of the Seventh International Symposium on Aviation Psychology (pp. 1-11). Columbus, OH: Department of Aviation, The Ohio State University.

5. Schutte, P. Complemation: An alternative to automation. Journal of Information Technology Impact, 1(3), (1999): 113-118.

6. Cook, Catherine A., et al. "Function allocation for future systems: Developing an improved methodology." Proceedings of the Human Factors and Ergonomics Society Annual Meeting. Vol. 40. No. 19. SAGE Publications, 1996. 
7. Flemisch, F. O., Adams, C. A., Conway, S. R., Goodrich, K. H., Palmer, M. T., \& Schutte, P. C. (2003). The H-metaphor as a guideline for vehicle automation and interaction. (Technical Memorandum No. NASA/TM-2003-212672). Hampton, VA: NASA Langley Research Center.

8. Schutte, P. C., Goodrich, K. H., et al, 2007, “The Naturalistic Flight Deck System: An Integrated System Concept for Improved Single-Pilot Operations," NASA/TM-2007-21509. Washington, DC.: National Aeronautics and Space Administration.

9. Goodrich, K.H., Schutte, P.C. and Williams, R.A, 2011, Haptic-Multimodal Flight Control System Update, 11th AIAA Aviation Technology, Integration, and Operations Conference (ATIO), Virginia Beach, VA.

10.Kanki, B., Helmreich, R., \& Anca, J. (Eds.) Crew Resource Management, Academic Press, San Diego, CA, 2010.

11.Dismukes, R. K., and J. L. Nowinski. "Prospective memory, concurrent task management, and pilot error." Attention: From theory to practice (2006): 225-236.

12.Schutte, P. C., Goodrich, K.H., \& Williams, R. A., Towards an Improved Pilot- Vehicle Interface for Highly Automated Aircraft: Evaluation of the Haptic Flight Control System, Proceedings of 4th International Conference on Human Factors and Ergonomics, July 2012.

13.Schutte, P. C., Goodrich, K.H., \& Williams, R. A., Evaluation of the Haptic Flight Control System: A Common Interface for Manual and Automatic Control Modes,

14.Hart, S. and Staveland, L. E., 1988, Human Mental Workload, Development of NASA TLX: Results of Empirical and Theoretical Research. North Holland Press, Amsterdam.

15.Endsley, M.R., 1988, Situation Awareness Global Assessment Technique (SAGAT). In Proceedings of the National Aerospace and Electronics Conference (NAECON) (pp. 789-795). New York: IEEE. 